\title{
Macroinvertebrados bentónicos de ríos de zonas áridas del noroeste argentino
}

\author{
Guillermo E. Hankel ${ }^{1}$, Daniel Emmerich ${ }^{1}$ \& Carlos Molineri $^{1, \bigotimes}$ \\ ${ }^{1}$ Instituto de Biodiversidad Neotropical, Facultad de Ciencias Naturales, CONICET-UNT. Tucumán, Argentina.
}

\begin{abstract}
RESUMEN. Una gran parte del noroeste argentino (NOA) está ocupada por zonas áridas vulnerables al avance de las actividades humanas. En este trabajo se caracterizaron los ensambles de macroinvertebrados de los ríos de esas zonas, para lo cual se emplearon distintos índices bióticos a fin de evaluar su respuesta y posible aplicación en la región. En las provincias de Catamarca y La Rioja fueron muestreados 15 ríos y arroyos, en diferentes períodos (23 sitios en total). Se tomaron muestras Surber (dos o tres por sitio) y se registraron datos ambientales. Se realizaron análisis de correspondencia canónica (CCA) para estudiar las relaciones entre los datos ambientales y los ensambles de macroinvertebrados, y se aplicaron diferentes índices bióticos. En total fueron identificados 59 taxa, de los cuales Diptera fue el más rico (12 familias). La máxima diversidad alfa encontrada correspondió a 29 taxa, mientras que la mínima fue 1. Los taxa más abundantes fueron Andesiops peruvianus, Meridialaris tintinnabula, Austrelmis (larva), Orthocladiinae, Smicridea, Helicopsyche y Heleobia. El CCA reveló que la conductividad eléctrica y el ancho húmedo son factores clave que influyen en la estructura de los ensambles de macroinvertebrados. Entre los índices bióticos utilizados, el de las Sierras de San Luis (IBSSL) mostró la mayor concordancia con el estado ecológico de los ambientes estudiados. Algunos de los sitios investigados poseen una diversidad similar a sitios de zonas áridas de la Argentina, como Cuyo (provincias de San Luis y Mendoza) y la provincia de Catamarca, pero de menor riqueza que los de Patagonia. Los resultados muestran que el IBSSL se puede usar en la región, pero aún deben realizarse ajustes en los valores de corte, tanto para este índice como para otros empleados. Por esta razón, y para mejorar su valor explicativo, se requiere de estudios más detallados que incluyan sitios impactados en diferente grado.
\end{abstract}

[Palabras clave: ambientes áridos, ecorregión Monte, Catamarca, La Rioja, arroyos, índices bióticos]

Aвstract. Benthic macroinvertebrates of arid environments of northwestern of Argentina. In the Northwestern region of Argentina (NOA), an extensive part of the territory is occupied by arid environments, vulnerable to the development of human economic activities. The aim of this study was to provide basic information about the benthic macroinvertebrate assemblages of rivers in this area, and to use different biotic indices to prove their power to predict river ecological status. Fifteen streams and rivers were sampled in the provinces of Catamarca and La Rioja, in different occasions, accounting for a total of 23 sites. Samples were taken with Surber net (two or three in each site) and environmental parameters were recorded. Canonical correspondences analyses (CCA) were performed to study the relationships between environmental data and the macroinvertebrate assemblage structure, and different biotic indices were calculated for each site. A total of 59 taxa were found, being Diptera the richest group (12 families). The highest alfa diversity was 29 taxa, while the lowest was 1 . The best represented taxa were Andesiops peruvianus, Meridialaris tintinnabula, Austrelmis (larva), Orthocladiinae, Smicridea, Helicopsyche and Heleobia. The CCA showed that electric conductivity and streambed width were important factors that influence the macroinvertebrate assemblage structure. The biotic index with the best ecological performance was the "índice biótico de las Sierras de San Luis" (IBSSL). Diversity in the studied sites was similar to those of other sites from arid environments of Argentina, like Cuyo (San Luis and Mendoza provinces) and Catamarca province, but lower than those of the Patagonian sites. The IBSSL proved to be useful to be applied in the region, but more detailed studies are needed, including polluted sites to improve their explanatory value.

[Keywords: aridity, Monte ecoregion, Catamarca province, La Rioja province, streams and rivers]

Editora asociada: Irina Izaguirre $\bar{\triangle}$ carlosmolineri@gmail.com
Recibido: 25 de agosto de 2017

Aceptado: 16 de marzo de 2018 


\section{INTRODUCCIÓN}

En la Argentina, las zonas áridas o semiáridas forman una gran extensión territorial, por lo que constituyen uno de sus ambientes principales. Ocupan gran parte del noroeste del país (NOA, noroeste argentino), así como las regiones de Cuyo y Patagonia. En estos ambientes, los ríos y arroyos son bienes públicos clave que se ven amenazados por el avance de actividades de gran impacto sobre los ecosistemas (e.g., la megaminería hidrogeoquímica metalífera y uranífera, y el monocultivo de soja). Los macroinvertebrados bentónicos en estos sitios han recibido diferente atención, y los más estudiados son los ambientes de Patagonia y Cuyo (e.g., Miserendino 2001; Miserendino and Brand 2007; Pessaq and Miserendino 2008 para Patagonia; Medina et al. 1997; Medina and Paggi 2004; Scheibler and Debandi 2008; Scheibler et al. 2008 para Cuyo). En las zonas áridas del NOA hay estudios para las regiones Monte, Chaco y Puna (e.g., Tejerina and Molineri 2007; Colla et al. 2012; Zelarayán and Salas 2012; Nieto et al. 2017), aunque existe un gran vacío de información de base en regiones amenazadas.

Los macroinvertebrados son indicadores fundamentales de la calidad de los ambientes acuáticos porque son diversos taxonómica y ecológicamente, tienen ciclos de vida relativamente largos y el muestreo es sencillo y poco oneroso. Además, poseen una sistemática sólida en los niveles de familia y género (Prat et al. 2009). El uso de índices bióticos basados en macroinvertebrados como medida de evaluación de la calidad ecológica del agua está bastante extendido, tanto en el mundo (e.g., Alba-Tercedor et al. 2004) como en Argentina (Domínguez and Fernández 1998; Pizzolon and Miserendino 2001; Pave and Marchese 2005; Fernández et al. 2006; Miserendino et al. 2008) y particularmente para nuestra región (e.g., Figueroa et al. 2003). Sin embargo, la aplicación de índices bióticos para la evaluación de la calidad del agua requiere de un buen conocimiento de base de la biota bentónica (Mesa 2011).

El presente estudio tiene como objetivo presentar un relevamiento de macroinvertebrados acuáticos y variables ambientales en ríos de zonas áridas del NOA, que pueden ser tomados como sitios de referencia para posteriores estudios de diversidad y bioindicación en la zona. Empleamos índices bióticos para observar su desempeño en estos ríos.

\section{Materiales y Métodos}

\section{Área de estudio}

Corresponde a las provincias de Catamarca y La Rioja en el NOA (Figura 1). El clima es árido y se lo clasifica como tipo B en el esquema de Köppen (climas con déficit hídrico), con precipitaciones anuales entre 100 y $400 \mathrm{~mm}$ (Rosa 2000; Bravo et al. 2001). Posee una continentalidad marcada que se manifiesta en una gran amplitud térmica, en especial durante el verano. Las precipitaciones se concentran en los meses de verano (70\%) y se manifiestan generalmente como tormentas o aguaceros. En varios meses del año se registran eventos de heladas frecuentes (Parra and Morales 2003). En términos biogeográficos, se ubica en las regiones Neotropical (Cabrera and Willink 1973) y de Transición Sudamericana (Morrone 2004), y se ubica en las ecorregiones de Altos Andes, Puna, Prepuna, Monte y Chaco (Brown et al. 2006).

\section{Muestreo y procesamiento de las muestras}

Se muestrearon en total 15 ríos y arroyos (Figura 1, Tabla 1) entre los años 2005 y 2013. Ocho de estos sitios se muestrearon dos veces (en aguas altas y aguas bajas), mientras que el río Santa María fue muestreado en 3 sitios distintos, totalizando 23 eventos muestrales. En su mayoría, los sitios escogidos corresponden a ríos sin perturbaciones de importancia, por lo que podrían considerarse como no impactados o de referencia. Pueden representar una excepción a esto los sitios COLO (por ser un arroyo aislado, de origen termal, que se encuentra canalizado; fue muestreado cerca de una toma de agua con actividad humana en su ribera) y NACI (Figura 2 a y b) (por estar ubicado cerca de la Mina Gualcamayo, provincia de San Juan, de la que recibe el polvillo de las explosiones; fue muestreado aguas abajo de un azud en desuso). Por otra parte, los sitios CUEV y RUBI (Figura 2 c y d) corresponden a arroyos prístinos de altura. El sitio MIRI, corresponde al río Miranda (de montaña, con tamaño mediano y sin sombra en sus riberas). El sitio OLTA es un arroyo de montaña con mucha pendiente; recorre una cañada honda sin impacto humano visible. En el momento del muestreo se encontraba casi sin caudal, con una baja velocidad de corriente, y su cauce se interrumpía por trechos; por lo tanto, las muestras se tomaron en los pozones y exiguas correderas que permanecían con agua. Los sitios restantes, desde NAC1 hasta SMFA, están ubicados en la provincia de Catamarca, 
Tabla 1. Sitios de muestreo y parámetros ecológicos y físico-químicos registrados. Los sitios corresponden a: Colorado (COLO), Olta (OLTA), Los Nacimientos (NACI), Miranda invierno (MIRI), Las Cuevas (CUEV), La Rubia (RUBI), Nacimiento 1 (NAC1), Nacimiento 2 (NAC2) (14), Santa María en Corral Viejo 1 (SMC1), Santa María en Corral Viejo 2 (SMC2), El Tesoro 1 (TES1), El Tesoro 2 (TES2), Las Cuevas 1 (CUE1), Las Cuevas 2 (CUE2), El Ingenio 1 (ING1), El Ingenio 2 (ING2), Dos Ríos2 (DOS2), Las Conchas 1 (CON1), Las Conchas 2 (CON2), Blanco 1 (BLA1), Blanco 2 (BLA2), Santa María en Agua de las Palomas 2 (SMAP), Los Corrales (CORR), Santa María en Famabalasto (SMF). $1=$ aguas bajas; $2=$ aguas altas.

Table 1. Sampled sites, and ecological and physical-chemical parameters recorded. Sites are: Colorado (COLO) (1), Olta (OLTA) (2), Los Nacimientos (NACI) (5), Miranda invierno (MIRI) (6), Las Cuevas (CUEV) (10), La Rubia (RUBI) (11), Nacimiento 1 (NAC1) AB (13), Nacimiento 2 (NAC2)AA (14), Santa María en Corral Viejo 1 (SMC1)AB (15), Santa María en Corral Viejo 2 (SMC2)AA (16), El Tesoro 1 (TES1)AB (17), El Tesoro 2 (TES2)AA (18), Las Cuevas 1 (CUE1)AB (19), Las Cuevas 2 (CUE2)AA (20), El Ingenio 1 (ING1)AB (21), El Ingenio 2 (ING2)AA (22), Dos Ríos 2 (DOS2) AA (23), Las Conchas 1 (CON1)AB (24), Las Conchas 2 (CON2)(25), Blanco 1 (BLA1)AB (26), Blanco 2 (BLA2)AA (27), Santa María en Agua de las Palomas 2 (SMAP)AA (28), Los Corrales (CORR) (29), Santa María en Famabalasto (SMF) (30). 1=low water season; $2=$ high water season.

\begin{tabular}{|c|c|c|c|c|c|c|c|c|c|c|c|}
\hline 苛 & 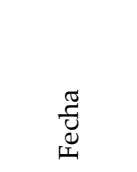 & 蔦 & 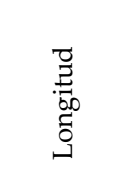 & 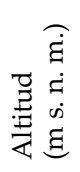 & 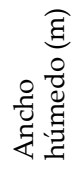 & 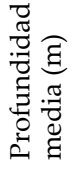 & $\frac{T_{2}}{2}$ & 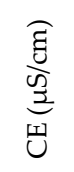 & 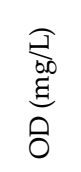 & 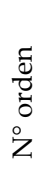 & 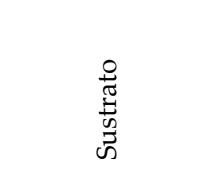 \\
\hline COLO & XII/2011 & -28.6492 & -66.5602 & 653 & 0.3 & 0.04 & 7.86 & 1690 & 7.34 & 3 & $\begin{array}{l}\text { Cascajo grueso / } \\
\text { arena gruesa }\end{array}$ \\
\hline OLTA & VIII/2013 & -30.6288 & -66.3292 & 660 & 0.5 & 0.10 & 7.95 & 260 & 9.73 & 3 & $\begin{array}{l}\text { Piedra fina / } \\
\text { cascajo grueso }\end{array}$ \\
\hline NACI & X/2009 & -29.5769 & -68.6636 & 1408 & 3.0 & 0.15 & 8.49 & 660 & 5.99 & 3 & $\begin{array}{l}\text { Piedra fina / } \\
\text { arena gruesa }\end{array}$ \\
\hline MIRI & VIII/2013 & -29.3479 & -67.7037 & 1514 & 2.0 & 0.25 & 7.80 & 340 & 10.38 & 4 & $\begin{array}{l}\text { Piedra fina / } \\
\text { cascajo fino }\end{array}$ \\
\hline CUEV & X/2009 & -28.9265 & -67.6763 & 2851 & 1.0 & 0.10 & 8.56 & 240 & 8.74 & 2 & $\begin{array}{c}\text { Piedra gruesa / } \\
\text { bloque }\end{array}$ \\
\hline RUBI & X/2009 & -28.9403 & -67.6764 & 2967 & 1.0 & 0.10 & 8.69 & 230 & 9.08 & 3 & $\begin{array}{c}\text { Piedra gruesa / } \\
\text { bloque }\end{array}$ \\
\hline NAC1 & $X / 2005$ & -27.1654 & -66.7449 & 2250 & 3.0 & 0.14 & 9.08 & 350 & 4.95 & 3 & $\begin{array}{l}\text { Piedra fina / } \\
\text { piedra gruesa }\end{array}$ \\
\hline NAC2 & II/2006 & -27.1654 & -66.7449 & 2250 & 5.0 & 0.09 & 8.42 & 506 & 7.57 & 3 & $\begin{array}{l}\text { Piedra fina / } \\
\text { piedra gruesa }\end{array}$ \\
\hline SMC1 & $X / 2005$ & -26.9860 & -66.2652 & 2415 & 2.0 & 0.07 & 8.49 & 530 & 7.70 & 5 & $\begin{array}{l}\text { Arena gruesa / } \\
\text { arena fina }\end{array}$ \\
\hline SMC2 & II/2006 & -26.9860 & -66.2652 & 2415 & 20.0 & 0.25 & 8.19 & 182 & 8.09 & 5 & $\begin{array}{c}\text { Arena gruesa / } \\
\text { arena fina }\end{array}$ \\
\hline TES1 & IX/2005 & -27.0923 & -66.1493 & 2820 & 1.0 & 0.07 & 8.62 & 300 & 4.94 & 4 & $\begin{array}{c}\text { Cascajo grueso / } \\
\text { piedra fina }\end{array}$ \\
\hline CUE1 & X/2005 & -28.9265 & -67.6763 & 2480 & 1.0 & 0.08 & 8.43 & 614 & 4.96 & 2 & $\begin{array}{l}\text { Arena gruesa / } \\
\text { bloque fino }\end{array}$ \\
\hline CUE2 & II/2006 & -28.9265 & -67.6763 & 2480 & 6.0 & 0.24 & 8.58 & 317 & 9.22 & 2 & $\begin{array}{c}\text { Arena gruesa / } \\
\text { bloque fino }\end{array}$ \\
\hline ING1 & X/2005 & -27.2266 & -66.2447 & 3000 & 1.0 & 0.08 & 8.25 & 130 & 4.95 & 2 & $\begin{array}{l}\text { Piedra fina / } \\
\text { cascajo grueso }\end{array}$ \\
\hline ING2 & II/2006 & -27.2266 & -66.2447 & 3000 & 1.8 & 0.16 & 8.10 & 100 & 9.40 & 2 & $\begin{array}{l}\text { Piedra fina / } \\
\text { cascajo grueso }\end{array}$ \\
\hline DOS2 & II/2006 & -27.2277 & -66.2125 & 3180 & 0.9 & 0.07 & 8.32 & 239 & 8.71 & 2 & $\begin{array}{c}\text { Cascajo grueso / } \\
\text { piedra gruesa }\end{array}$ \\
\hline CON1 & X/2005 & -27.2808 & -66.2697 & 3190 & 0.5 & 0.07 & 8.10 & 320 & 4.96 & 3 & $\begin{array}{c}\text { Cascajo grueso / } \\
\text { piedra fina }\end{array}$ \\
\hline CON2 & II/2006 & -27.2808 & -66.2697 & 3190 & 1.0 & 0.08 & 8.43 & 290 & 10.00 & 3 & $\begin{array}{c}\text { Cascajo grueso / } \\
\text { piedra fina }\end{array}$ \\
\hline BLA1 & IX/2005 & -27.3095 & -66.3119 & 3135 & 0.5 & 0.05 & 7.98 & 390 & 5.03 & 3 & $\begin{array}{c}\text { Cascajo grueso / } \\
\text { piedra fina }\end{array}$ \\
\hline BLA2 & II/2006 & -27.3095 & -66.3119 & 3135 & 0.8 & 0.15 & 8.21 & 330 & 12.79 & 3 & $\begin{array}{l}\text { Cascajo grueso / } \\
\text { piedra fina }\end{array}$ \\
\hline SMAP & II/2006 & -26.9851 & -66.1589 & 2350 & 22.0 & 0.30 & 8.11 & 188 & 8.28 & 5 & $\begin{array}{l}\text { Arena gruesa / } \\
\text { piedra gruesa }\end{array}$ \\
\hline CORR & II/2006 & -27.3593 & -66.3622 & 3180 & 0.5 & 0.05 & 8.33 & 860 & 5.05 & 2 & $\begin{array}{l}\text { Arena gruesa / } \\
\text { cascajo fino }\end{array}$ \\
\hline SMFA & II/2006 & -26.9604 & -66.2818 & 2215 & 20.0 & 0.20 & 8.24 & 159 & 9.18 & 5 & $\begin{array}{c}\text { Arena gruesa / } \\
\text { piedra gruesa }\end{array}$ \\
\hline
\end{tabular}




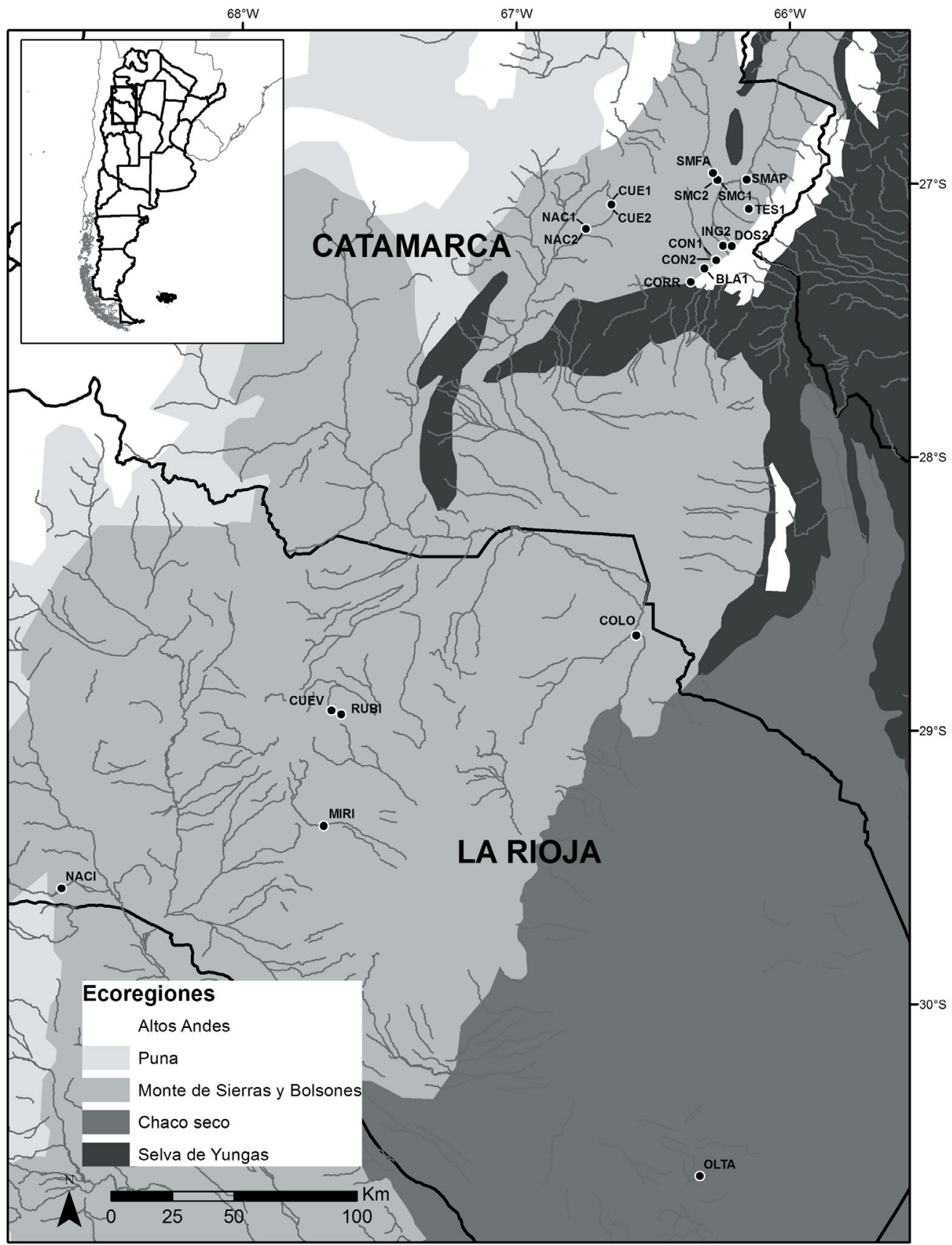

Figura 1. Ubicación de los sitios muestreados en las provincias de Catamarca y La Rioja, Argentina. Los códigos para los sitios son los mismos mostrados en la Tabla 1.

Figure 1. Sampled sites in Catamarca and La Rioja provinces, Argentina. Codes for the sites are the same showed in Table 1.

en la zona conocida como Campo del Arenal. Son arroyos de orden bajo (excepto el río Santa María), con sustrato arenoso y una hidrología de alta estacionalidad.

Las muestras se recolectaron con red Surber (30x30 cm, 300 um de apertura de malla), dos o tres por sitio, y se fijaron con etanol $96^{\circ}$. Se tomaron datos de altitud, ancho húmedo, profundidad, temperatura del agua, $\mathrm{pH}$, conductividad eléctrica (CE) y oxígeno disuelto (OD) (Tabla 1). Los datos físicos y químicos se obtuvieron con un analizador multiparamétrico marca HORIBA, modelo U52. La granulometría del sustrato fue tomada utilizando una ruleta de $25 \mathrm{~m}$ ubicada en distintas direcciones (3 repeticiones) sobre el lecho (incluyendo el húmedo y el seco), y 


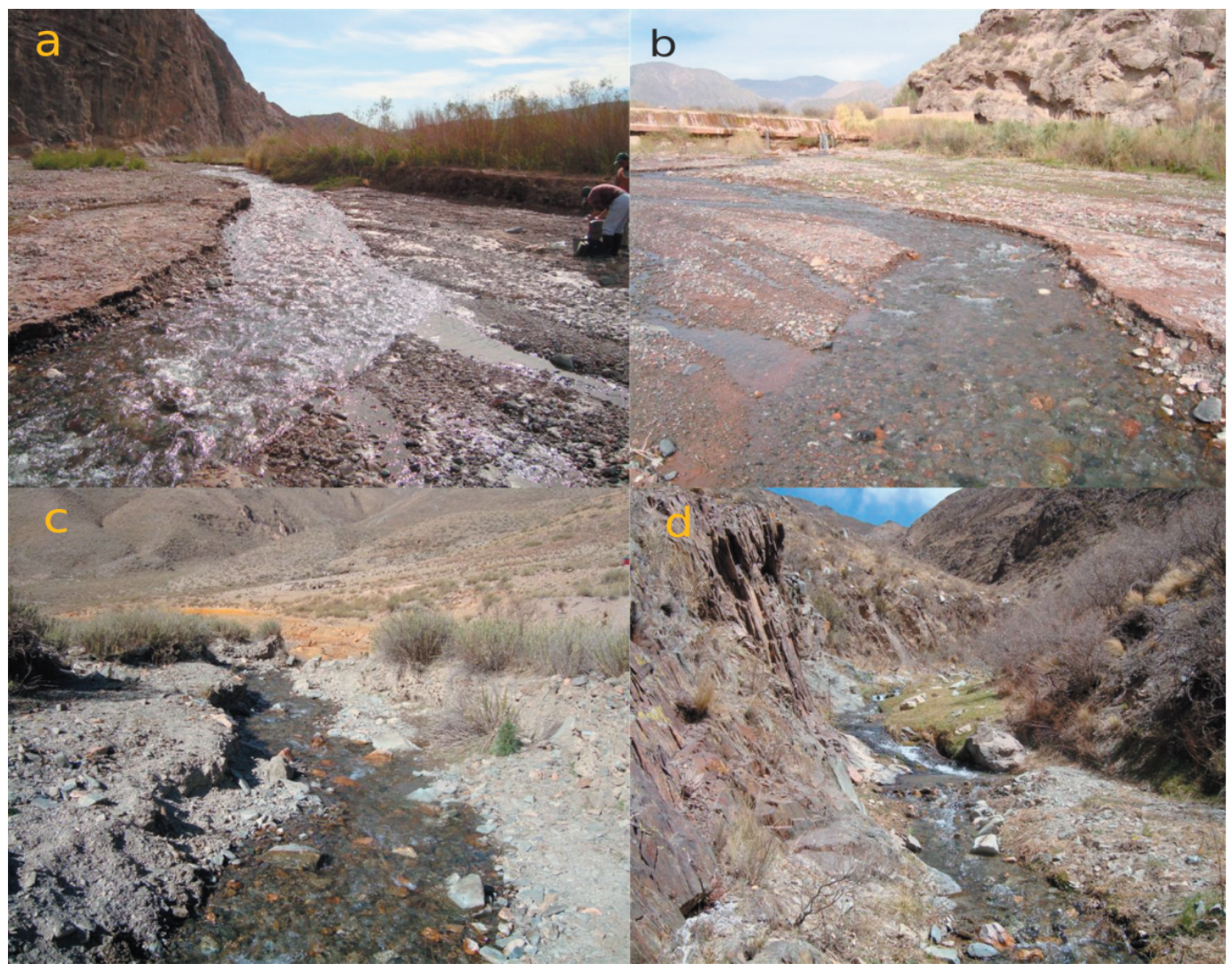

Figura 2. Algunos de los sitios muestreados en la provincia de La Rioja, Argentina. a) y b) Arroyo Los Nacimientos, con el detalle del azud en desuso en el arroyo (imagen b). c) Arroyo Las Cuevas. d) Arroyo La Rubia.

Figure 2. Some sampled sites in La Rioja province, Argentina. a) y b) Los Nacimientos stream, with a view of the disused dam in the stream (image b). c) Las Cuevas stream. d) La Rubia stream.

se registró el sustrato presente cada $0.5 \mathrm{~m}$. A partir de estos datos se calculó el porcentaje de cada clase de tamaño (en $\mathrm{mm}$ ): bloque (>256), piedra gruesa (192-256), piedra fina (64 a 192), cascajo grueso (32 a 64), cascajo fino (16 a 32), grava gruesa (9 a 16), grava fina ( 2 a 9), arena gruesa (1 a 2$)$, arena fina $(0.06$ a 1$) \mathrm{y}$ limo-arcilla $(<0.06)$ (modificado de Cummins 1962).La jerarquía de los cauces (número de orden) fue calculada según la escala propuesta por Sthraler (Welcome 1985).

En el laboratorio, las muestras fueron procesadas en su totalidad bajo lupa estereoscópica a 10 y 20X; el material orgánico y el mineral se desecharon y los organismos fueron separados y conservados en alcohol $96^{\circ}$. Los macroinvertebrados fueron identificados hasta el nivel de especie para Ephemeroptera, Odonata y Gripopterygidae (Plecoptera) y hasta género para los restantes grupos (excepto en el caso de Diptera, cuya resolución taxonómica fue hasta el nivel de familia). Se emplearon claves de identificación generales (Domínguez and Fernández 2009; Salcedo Gustavson and Trama 2014) y específicas (von Ellenrieder and Garrison 2007; Molineri 2010a). Las muestras se encuentran depositadas en la colección del Instituto de Biodiversidad Neotropical (IBN).

\section{Tratamiento de los datos}

Los sitios se codificaron con cuatro letras relacionadas a su nombre $y$, en caso de que fuera pertinente, los números 1 ó 2 para indicar si corresponde a aguas bajas o altas, respectivamente. Las muestras Surber fueron promediadas y expresadas como individuos/ $\mathrm{m}^{2}$ (Apéndice 1). Se calcularon los índices de diversidad de Shannon $\left(\mathrm{H}^{\prime}\right)$ y Simpson (éste es un índice de dominancia, por lo cual la diversidad se expresa como 1-D, donde D es el valor del índice) y la equitatividad o equidad ( $\mathrm{J}^{\prime}$, también llamado índice de Pielou) mediante la fórmula $\mathrm{J}^{\prime}=\mathrm{H}^{\prime} / \ln (\mathrm{S})$, donde $\mathrm{H}^{\prime}$ es el índice de Shannon y S es la riqueza taxonómica. Para evaluar las relaciones entre las variables ambientales y la estructura del ensamble de macroinvertebrados se emplearon análisis de correspondencia canónica (CCA), cuya significancia se evaluó 
mediante la prueba de Montecarlo. Debido a la naturaleza de los datos con los que contamos (algunos sitios tienen repeticiones y otros no, lo que podría distorsionar el análisis al poseer igual valor para la variable altitud), se realizó el ordenamiento incluyendo y sin incluir la variable altitud. Los resultados de ambos mostraron un patrón similar, por lo que se optó por presentar el análisis incluyendo dicha variable. Tanto los CCA como el cálculo de los índices de diversidad (excepto J') se hicieron con el paquete vegan (Oksanen et al. 2016) del software estadístico R (R Core Team 2016). Los datos ambientales y biológicos (excepto el pH) se estandarizaron por medio de la fórmula " $\ln (\mathrm{x}+1)$ ". Se calcularon los siguientes índices bióticos: riqueza taxonómica $(S$, calculado como la suma de los taxa encontrados en un sitio), BMWP' y ASPT' (adaptados por Domínguez y Fernández [1998]), índice biótico de Yungas (IBY4) (Dos Santos et al. 2011), número de taxa de EphemeropteraPlecoptera-Trichoptera (EPT), número de taxa de Elmidae, Plecoptera y Trichoptera (ElPT) (von Ellenrieder 2007) e índice biótico de las Sierras de San Luis (IBSSL) (Vallania et al. 1996). Se consideraron sólo dos estados ecológicos para los ríos evaluados: impactado o no impactado, y los valores de corte para distinguir el estado ecológico mencionado son los enunciados en Dos Santos et al. (2011) y Vallania et al. (1996).

\section{Resultados}

\section{Comunidades bentónicas}

En la Tabla 1 se indican los sitios muestreados, sus códigos y las variables ambientales medidas. En el Apéndice 1 se encuentra el listado completo de abundancia (individuos/ $\mathrm{m}^{2}$ ) de los taxones por sitio. Se identificaron en total 59 taxa (Apéndice 1). La mayor riqueza se encontró en los sitios MIRI, SMC1, BLA2 y CORR con 29, 26, 25 y 25 taxa, respectivamente, mientras que los sitios NAC2, SMC2 y CUE2 presentaron la menor riqueza (un taxón cada uno) (Tabla 2). Estos últimos tres sitios también presentaron una abundancia muy baja (menos de 8 individuos), probablemente a causa de que el muestreo se realizó justo después de crecidas estacionales importantes y a que poseen un lecho arenoso, lo que favorece la baja diversidad de los macroinvertebrados (los muestreos cualitativos no aportaron más taxones). Por su parte, los índices de diversidad señalaron a los sitios SMAP, CORR, MIRI y SMFA como los más diversos, mientras que los menores valores correspondieron siempre a los sitios NAC2, SMC2 y CUE2,

Tabla 2. Valores obtenidos para los índices bióticos, calculadose índices de diversidad. La codificación de los sitios es la misma que aparece en la Tabla $1 . \mathrm{H}^{\prime}$ es el índice de Shannon-Wiener. 1-D es el índice de Simpson. J' es el índice de Pielou (equitatividad). * denota un sitio calificado como "no impactado".

Table 2. Scores for biotic indices. Site codes are the same employed in Table 1. H' is Shannon-Wiener's index. 1-D is Simpson's index. J' is Pielou index (evenness). *indicate sites classified as "not impacted".

\begin{tabular}{|c|c|c|c|c|c|c|c|c|c|c|}
\hline Sitios & IBSSL & EPT & ElPT & IBY 4 & Riqueza & $\mathrm{BMWP}^{\prime}$ & $\mathrm{ASPT}^{\prime}$ & $\mathrm{H}^{\prime}$ & $1-\mathrm{D}$ & $\mathrm{J}^{\prime}$ \\
\hline COLO & 8 & 3 & $5^{*}$ & $3^{*}$ & $16^{*}$ & 49 & 5.44 & 1.64 & 0.68 & 0.59 \\
\hline OLTA & 8 & 7 & $7^{*}$ & 2 & $21^{*}$ & $73^{*}$ & 4.75 & 2.00 & 0.82 & 0.66 \\
\hline NACI & 8 & 5 & 2 & 1 & 14 & 39 & 4.33 & 1.16 & 0.58 & 0.44 \\
\hline MIRI & 9 & 8 & $7^{*}$ & $3^{*}$ & $29^{*}$ & $112^{*}$ & 5.09 & 2.19 & 0.84 & 0.65 \\
\hline CUEV & 9 & 6 & $6^{*}$ & $3^{*}$ & $22^{*}$ & $86^{*}$ & 5.06 & 1.66 & 0.68 & 0.54 \\
\hline RUBI & 9 & 5 & $5^{*}$ & $3^{*}$ & $23^{*}$ & $74^{*}$ & 4.63 & 1.61 & 0.69 & 0.51 \\
\hline NAC1 & 10 & 3 & 3 & 2 & $19^{*}$ & 53 & 3.79 & 1.89 & 0.78 & 0.64 \\
\hline NAC2 & 4 & 0 & 0 & 0 & 1 & 5 & 5.00 & 0.00 & 0.00 & 0.00 \\
\hline SMC1 & 11 & 5 & 4 & 2 & $26^{*}$ & $87^{*}$ & 4.35 & 1.48 & 0.63 & 0.45 \\
\hline SMC2 & 4 & 0 & 0 & 0 & 1 & 3 & 3.00 & 0.00 & 0.00 & 0.00 \\
\hline TES1 & 11 & 4 & $5^{*}$ & $3^{*}$ & $22^{*}$ & $72^{*}$ & 4.50 & 1.54 & 0.66 & 0.50 \\
\hline CUE1 & 11 & 5 & $5^{*}$ & 2 & $22^{*}$ & $67^{*}$ & 4.19 & 1.71 & 0.69 & 0.55 \\
\hline CUE2 & 4 & 0 & 1 & 1 & 1 & 5 & 5.00 & 0.00 & 0.00 & 0.00 \\
\hline ING1 & 11 & 5 & 4 & 2 & $21^{*}$ & $73^{*}$ & 4.56 & 2.06 & 0.81 & 0.68 \\
\hline ING2 & 8 & 4 & 2 & 1 & 10 & 44 & 4.89 & 1.36 & 0.62 & 0.59 \\
\hline DOS2 & 9 & 3 & 2 & 2 & 12 & 40 & 5.00 & 0.93 & 0.43 & 0.37 \\
\hline CON1 & 11 & 6 & $5^{*}$ & $3^{*}$ & $23^{*}$ & $87^{*}$ & 4.83 & 1.90 & 0.70 & 0.61 \\
\hline CON2 & 4 & 0 & 0 & 0 & 4 & 11 & 3.67 & 1.13 & 0.59 & 0.82 \\
\hline BLA1 & 11 & 7 & $7^{*}$ & $3^{*}$ & $25^{*}$ & $83^{*}$ & 4.37 & 1.96 & 0.73 & 0.61 \\
\hline BLA2 & 6 & 1 & 2 & 2 & 9 & 26 & 3.71 & 1.81 & 0.79 & 0.82 \\
\hline SMAP & 8 & 3 & 3 & 2 & 6 & 27 & 5.40 & 2.64 & 0.70 & 1.47 \\
\hline CORR & 11 & 5 & $6^{*}$ & $3^{*}$ & $25^{*}$ & $90^{*}$ & 4.50 & 1.41 & 0.91 & 0.44 \\
\hline SMFA & 6 & 1 & 0 & 0 & 3 & 19 & 4.75 & 0.95 & 0.57 & 0.86 \\
\hline
\end{tabular}


que son los que presentan un solo taxón cada uno. Los taxa identificados fueron principalmente insectos (Apéndice 1); otros individuos observados correspondieron a los grupos Planariidae, Collembola, Crustacea, Hidracarina, Gastropoda y Oligochaeta. El grupo más diverso fue Diptera, con 14 taxa (mayormente familias), seguido por Trichoptera y Ephemeroptera, con 10 taxa (género o especie) cada uno. Se identificaron un total de 13700 individuos. Los ensambles de macroinvertebrados bentónicos resultaron distintos y diversos. Considerando la composición y estructura del ensamble, los sitios CUEV y RUBI mostraron la mayor similitud, con los efemerópteros Meridilaris tintinnabula Pescador y Peters 1987 y Andesiops peruvianus Ulmer 1920 representando la mayor proporción numérica, aunque alternando su dominio (M. tintinnabula domina en CUEV y A. peruvianus en RUBI), e incluyendo taxa exclusivos como Claudioperla tigrina Klapálek 1904 y Athericidae. Se destacan los sitios NAC2, CUE2 y SMC2, ya que presentaron muy bajas riqueza y abundancia (sólo un taxón en cada sitio). Mientras tanto, el sitio SMFA se encontró en una situación similar, pero con tres taxa (Muscidae, Orthocladiinae y Lachlania Hagen 1868). El sitio COLO fue el de mayor conductividad eléctrica $(1690 \mu \mathrm{S} / \mathrm{cm})$ y mostró una dominancia amplia de Smicridea McLachlan 1871, secundado por Heleobia Stimpson 1865 y larvas de Austrelmis Brown 1984. Los sitios OLTA y NACI presentaron una estructura distinta; estuvieron dominados por Helicopsyche von Siebold 1856 y Austrelmis adultos (OLTA), y por Heleobia y Baetodes huaico Nieto 2004 (NACI). El resto de los sitios no mostraron un patrón claro. Los taxa comunes en esos sitios fueron Orthocladiinae, $A$.

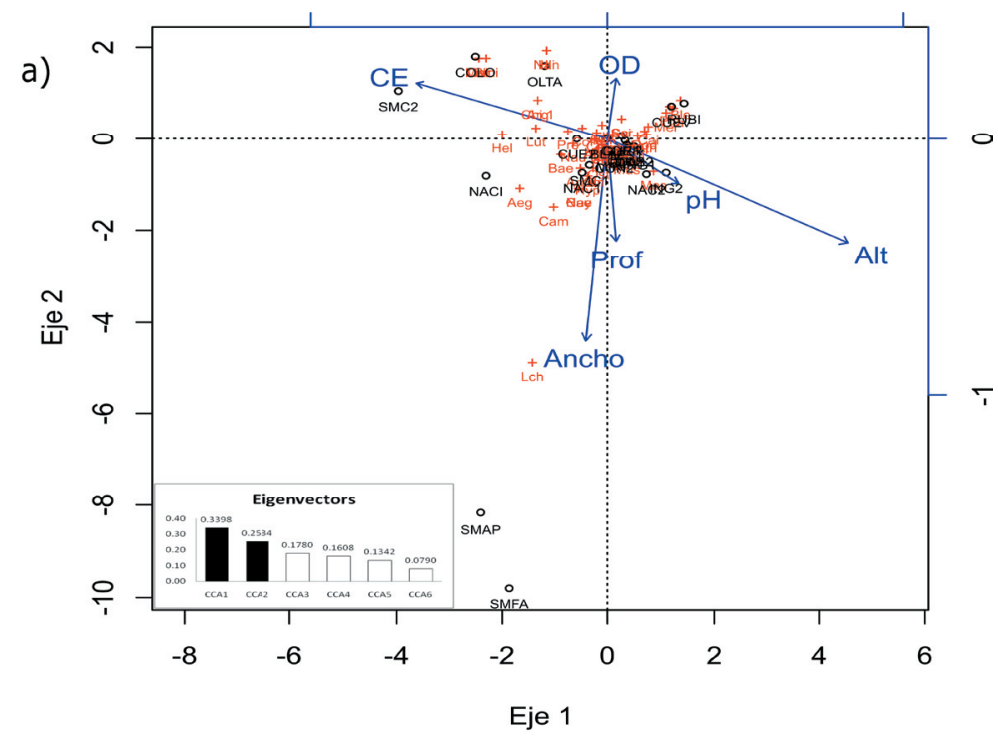

b)

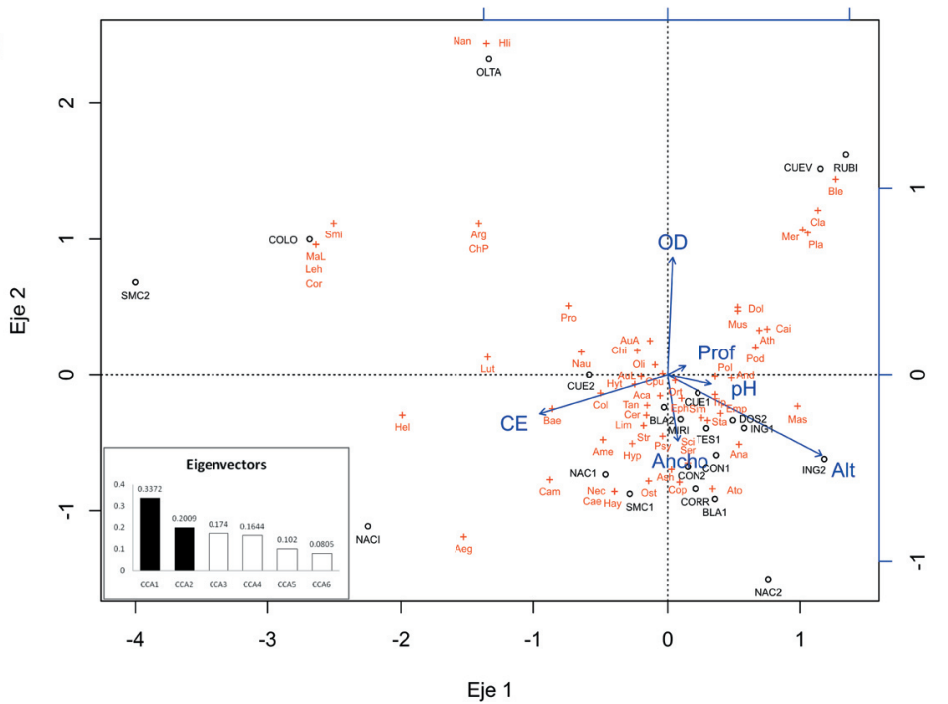

Figura 3. Triplot de los análisis de correspondencia canónica realizados. a) Gráfico que incluye todos los sitios en el análisis. b) Gráfico del análisis en el que se excluyeron los sitios SMAP y SMFA. Los sitios están representados por círculos vacíos $(\mathrm{O})$ y los taxa por cruces rojas (+). Las variables ambientales se representan por flechas azules. Las abreviaturas son las mismas que en la Tabla 1 (sitios) y Apéndice 1 (taxa). Vea el texto para una mejor explicación.

Figure 3. Canonical correspondenceanalysistriplot. a) Plot of the CCA including all sites sampled. b) Plot of CCA excluding the sites SMAP and SMFA. Sites are represented by circles $(\mathrm{O})$ and taxa by crosses $(+)$. Environmental variables are represented by arrows. Abbreviations are the same employed in the Table 1 (sites) and Appendix 1 (taxa). See text for a better explanation. 
peruvianus, Austrelmis (larvas y adultos), Ceratopogonidae, Staphylinidae, Simuliidae y Tipulidae. El CCA realizado explicó en los dos primeros ejes $44 \%$ de la variabilidad total y resultó significativo (prueba de Montecarlo, $P<0.001$ ). En el gráfico de este análisis (Figura 3 a), la mayor cantidad de sitios quedó ordenada muy cerca del origen de coordenadas; los sitios SMAP y SMFA se ubicaron en el extremo negativo del eje 2, asociados al ancho del lecho y a la presencia de Lachlania. Ante la dificultad de interpretar este gráfico, se realizó un nuevo CCA, sin estos dos sitios, que recogió $49 \%$ de la variabilidad y presentó un patrón similar en la distribución de los sitios, pero más espaciados, lo que facilitó su interpretación. En este análisis (Figura 3b), los sitios se ordenaron principalmente por su componente biológico. En el cuadrante positivo, separados de la nube de sitios, se encuentran los sitios CUEV y RUBI, mientras que en el cuadrante positivo del eje 1 y en el negativo del eje 2 están los sitios OLTA, COLO y SMC2, y el sitio NACI en el cuadrante negativo. Estos sitios poseen una estructura de la comunidad asociada que explica su separación en este ordenamiento. Los demás sitios se ubican cerca del origen, ya que poseen un grupo de especies en común.

\section{Índices bióticos}

En general, el índice que más coincidió con los valores de riqueza y diversidad fue el IBSSL (Tabla 2), que calificó como contaminados a aquellos sitios con muy baja diversidad y abundancia, y como en buen estado a los sitios restantes. En el empleo de este índice, los sitios NAC2, CUE2 y SMC2 quedaban sin valor asignado, ya que sólo contaban con una unidad taxonómica que no asigna valor numérico. Para subsanar este detalle se empleó el valor de la siguiente columna (como si tuviera entre 2 y 5 unidades taxonómicas) porque pensamos que es un arreglo que brinda una mejor resolución en vez de no asignar un valor. Los índices EPT y ASPT' calificaron a todos los sitios como impactados, mientras que los índices ElPT, IBY4, BMWP' y Riqueza tuvieron entre ellos un comportamiento similar, calificando cada uno a los mismos sitios como contaminados o en buen estado (Tabla 2).

\section{Discusión}

\section{Comunidades bentónicas}

Los sitios estudiados poseen una diversidad similar a la registrada en otros ambientes de la provincia de San Luis (Medina et al. 1997), mayor que los de Mendoza (Scheibler and Debandi 2008), Catamarca (Zelarayán and Salas 2007; Colla et al. 2012) y la Puna (Nieto et al. 2017), y menor que los de Patagonia (Miserendino 2001). Cabe señalar que no se descarta cierta subestimación de la diversidad por el hecho de haber determinado algunos taxones a nivel de familia. Esto es especialmente importante en el caso de Chironomidae, que puede presentar un gran número de géneros, aunque este grupo fue identificado hasta subfamilia para disminuir estos inconvenientes.

Los sitios CUEV y RUBI presentan comunidades muy similares dominadas por A. peruvianus y $M$. tintinnabula, y también son importantes Chironominae y C. tigrina. La estructura del ensamble es similar a lo que Scheibler y Debandi (2008) encontraron en Mendoza para su estación de muestreo de mayor altura (2236 m s. n. m.), dominada por Andesiops, Massartellopsis Demoulin 1955, Orthocladiinae (Medina et al. 2008) y coleópteros (Staphylinidae y Elmidae), aunque sin plecópteros. Los sitios referidos registraron también Podonominae, una subfamilia de Chironomidae, que es considerado estenotérmico frío, y restringido a ambientes de altura (Tejerina and Molineri 2007; Medina et al. 2008; Scheibler et al. 2008).

Los sitios OLTA, COLO y NACI son los de menor altitud y presentan ensambles particulares de macroinvertebrados dominados por Elmidae o Smicridea, lo que podría deberse a las condiciones particulares en el momento del muestreo. La baja diversidad del sitio NACI se explicaría por la presencia de un pequeño dique de nivelación aguas arriba del sitio de muestreo. La regulación de un curso de agua tiene efectos negativos sobre la diversidad biótica en el arroyo receptor, ya que interrumpe el transporte de detrito y de materia orgánica, y altera el flujo natural del sistema (Medina et al. 1997). Además, el río presenta un lecho con subdominancia de arena, lo que contribuye a homogeneizar el sustrato; a su vez, esto reduce la diversidad (Miserendino 2001; Allan and Castillo 2007). Adicionalmente, el sitio recibe polvo liberado por la actividad minera en las cercanías (Mina Gualcamayo, San Juan). OLTA mostró una composición similar a la reportada en arroyos de San Luis (Medina et al. 1997), y la contribución proporcional de subfamilias de Chironomidae muestra que Chironominae y Orthocladiinae fueron similares en abundancia, lo que coincide con ríos de Yungas de baja altitud (Tejerina 
and Molineri 2007). El sitio COLO presentó mayor temperatura y conductividad que los otros sitios; su cauce canalizado exhibió bajo caudal y mucha actividad humana en los alrededores (basura), lo que podría explicar la baja cantidad de Ephemeroptera (sólo un ejemplar de Leptohyphes eximius). Los sitios ING1, MIRI, TES1, CUE1, BLA1, CON1, ING2, NAC1, SMC1, DOS2 y CORR fueron los más diversos y mostraron los valores más altos de riqueza y abundancia. En su mayoría, estos sitios presentan comunidades dominadas por Orthocladiinae, con aportes (dependiendo del sitio) de Austrelmis (larva), Hydroptilidae y Andesiops (Apéndice 1). Esta dominancia de la comunidad por parte de Chironomidae también se observa en otros ríos de Catamarca (Zelarayán and Salas 2007; Colla et al. 2012). Los sitios de menor diversidad y abundancia (BLA2, CON2, NAC2, SMC2, CUE2, SMFA y SMAP) corresponden a ríos de Catamarca, muestreados durante aguas altas, lo que podría explicarse por las condiciones hidrológicas, ya que se reportó que las crecidas disminuyen en gran medida la diversidad y la abundancia de los macroinvertebrados bentónicos (Molineri 2009, 2010b; Mesa 2010; Romero et al. 2011). De acuerdo con los resultados del CCA, la conductividad eléctrica y el ancho húmedo fueron los factores más importantes que influyeron en los ensambles de macroinvertebrados. Sin embargo, estos resultados deben tomarse como preliminares ante la baja cantidad de muestreos realizados y la ausencia de mediciones de variables importantes como la velocidad de corriente o el caudal.

\section{Bioindicación}

El comportamiento de los índices bióticos y de diversidad calculados para los diferentes sitios refleja con claridad la importancia del factor "riqueza" en el cálculo de cada uno de ellos. Ninguno de los sitios muestreados podría ser catalogado como fuertemente contaminado, ya que en este estudio direccionamos nuestros muestreos hacia ambientes en buen estado (excepciones: el sitio COLO, que se encuentra canalizado, y NACI, que está embalsado y con actividad minera en las cercanías). El hecho de que algunos índices señalen "contaminación" para algunos sitios probablemente refleje lo inadecuado de los mismos para la zona estudiada, pues en ella existen ríos de por sí poco diversos en tramos de menor altura y con lecho arenoso (móvil), sobre todo cuando son muestreados en época de crecidas. Otro es el caso de ríos de mayor altitud, en donde las comunidades están formadas por unas pocas especies adaptadas a esos ambientes extremos. Otra razón para que los índices marquen a la mayor parte de los sitios como contaminados se debe a que los valores de corte utilizados para varios de estos índices (valores que separan ríos impactados de sanos) fueron propuestos para una zona mucho más diversa (Yungas) (Dos Santos et al. 2011). Sería prioritario adecuar los valores de corte para los ecosistemas estudiados aquí. Por estos motivos, esperaríamos que un potencial índice a ser utilizado en el monitoreo de estos ecosistemas tenga en cuenta la diversidad biológica y las tipologías de ríos presentes en la zona. El IBSSL parece tener un mejor desempeño que los demás, pues calificó a los arroyos en correspondencia con lo observado en campo, lo que es esperable, ya que este índice fue diseñado para sitios similares a los aquí estudiados. Sin embargo, es necesario incluir datos de sitios contaminados en el área de estudio, así como estudios que contemplen la variación temporal de los ensambles de macroinvertebrados.

Considerando la problemática del agua en el mundo, y que los ambientes áridos y semiáridos se ven más comprometidos con el avance de las actividades económicas de alto impacto ambiental, se vuelve prioritario el desarrollo de proyectos de investigación tendientes a impulsar el conocimiento de sus ecosistemas acuáticos. De esta manera, las decisiones de manejo y conservación de este bien imprescindible no se tomarán en un marco de ignorancia de la biota y de sus relaciones con el ambiente.

AgradeCimiEntos. Agradecemos sinceramente a las asambleas ciudadanas de Famatina, Chilecito, La Rioja (capital) y Olta por el afecto y apoyo en las campañas de muestreos. Especialmente, deseamos manifestar nuestra admiración por la constante defensa de los Bienes Naturales ante las políticas de actividades extractivas como la megaminería a cielo abierto. Con claridad exponen su derecho a elegir qué proyectos productivos sostenibles se deberían desarrollar en sus paisajes. Con coraje y dignidad expresan que "el agua vale más que el oro" y que actividades destructivas como la minería no tienen ni tendrán la licencia social. Agradecemos a los revisores anónimos por sus valiosas sugerencias que permitieron mejorar el manuscrito. A Luciana Cristóbal por su ayuda con la Figura 1. 


\section{REFERENCIAS}

Alba-Tercedor, J., P. Jáimez-Cuellar, M. Álvarez, J. Avilés, N. Bonada, J. Casas, et al. 2004. Caracterización del estado ecológico de ríos mediterráneos ibéricos mediante el índice IBMWP (antes BMWP'). Limnética 21(3-4):175-182.

Allan, J. D., and M. M. Castillo. 2007. Stream Ecology. Segunda Edición. Springer. Dordrecht. The Netherlands.

Bravo, G., A. Bianchi, J. Volante, S. Alderete Salas, G. Sempronii, L. Vicini, M. Fernández, H. Lipshitz, and A. Piccolo. 2001. Regiones agroeconómicas del Noroeste Argentino. INTA.

Brown, A., U. Martínez Ortiz, M. Acerbi, and J. Corcuera (eds.). 2006. La Situación Ambiental Argentina 2005. Fundación Vida Silvestre Argentina. Buenos Aires, Argentina.

Cabrera, A. L., and A. Willink. 1973. Biogeografía de América Latina. Monografía №13, Serie Biología, OEA. Secretaría General de la OEA, Washington, D.C. Estados Unidos.

Colla, M. F., I. I. César, and L. B. Salas. 2013. Benthic insects of the El Tala River (Catamarca, Argentina): longitudinal variation of their structure and the use of insects to assess water quality. Braz J Biol 73(2):357-366.

Cummins, K. W. 1962. An evaluation of some techniques for the collection and analysis of benthic samples with special emphasis on lotic waters. The American Midland Naturalist 67(2):477-504.

Domínguez, E., and H. R. Fernández. 1998. Calidad de los ríos de la cuenca del Salí (Tucumán, Argentina) medida por un índice biótico. Serie Conservación de la Naturaleza 12. Fundación Miguel Lillo, San Miguel de Tucumán, Tucumán, Argentina.

Domínguez, E., and H. R. Fernández (eds.). 2009. Macroinvertebrados Bentónicos Sudamericanos. Sistemática y biología. Fundación Miguel Lillo, San Miguel de Tucumán, Tucumán, Argentina.

Dos Santos, D., C. Molineri, M. C. Reynaga, and C. Basualdo. 2011. Which index is the best to assess stream health? Ecological Indicators 11(2):582-589.

Fernández, H. R., E. Domínguez, F. Romero, and M. G. Cuezzo. 2006. La calidad del agua y la bioindicación en los ríos de montaña del Noroeste Argentino. Serie Conservación de la naturaleza 16. Fundación Miguel Lillo, San Miguel de Tucumán, Tucumán, Argentina.

Figueroa, R., C. Valdovinos, E. Araya, and O. Parra. 2003. Macroinvertebrados bentónicos como indicadores de calidad de agua del sur de Chile. Rev Chil Hist Nat 76:275-285.

von Ellenrieder, N. 2007. Composition and structure of aquatic insect assemblages of Yungas mountain cloud forest streams in NW Argentina. Rev Soc Entomol Argent 66(3-4):57-76.

von Ellenrieder, N., and R. W. Garrison. 2007. Dragonflies and Damselflies (Insecta: Odonata) of the Argentine Yungas: Species composition and identification. Scientific Reports N ${ }^{\circ}$ 7. Publisher Società Zoologica "La Torbiera", Italy.

Medina, A. I., E. A. Vallania, E. S. Tripole, and P. A. Garefs.1997. Estructura y composición del zoobentos de ríos serranos (San Luis). Ecología Austral 7:28-34.

Medina, A. I., and A. C. Paggi. 2004. Composición y abundancia de Chironomidae (Diptera) en un río serrano de zona semiárida (San Luis, Argentina). Rev Soc Entomol Argent 63(3-4):107-118.

Medina, A. I., E. E. Scheibler, and A. C. Paggi. 2008. Distribución de Chironomidae (Diptera) en dos sistemas fluviales ritrónicos (Andino-Serrano) de Argentina. Rev Soc Entomol Argent 67(1-2):69-79.

Mesa, L. M. 2010. Effect of spates and land use on macroinvertebrates community in Neotropical Andean streams. Hydrobiologia 641:85-95.

Mesa, L. M. 2011. Diversidad de macroinvertebrados bentónicos en la cuenca del río Lules. Pp. 137-148 en Fernández, H. R. y H. M. Barber (eds.). La Cuenca del río Lules. Una aproximación multidisciplinaria a su complejidad. EDUNT San Miguel de Tucumán, Tucumán, Argentina.

Miserendino, M. L. 2001. Macroinvertebrates assemblages in Andean Patagonian rivers and streams: environmental relationships. Hydrobiologia 444:147-158.

Miserendino, M. L., and C. Brand. 2007. Trichoptera assemblages and environmental features in a large arid Patagonian river. Fundamental and Applied Limnology. Archiv für Hydrobiologie 169(4):307-318.

Miserendino, M. L., C. Brand, and C. Y. Di Prinzio. 2008. Assessing urban impacts on water quality, benthic communities and fish in streams of the Andes Mountains, Patagonia (Argentina). doi: 10.1007/s1 1270-008-9701-4.

Molineri, C. 2010a. Las especies de Leptohyphidae (Ephemeroptera) de las yungas de Argentina y Bolivia: diagnosis, distribución y claves. Rev Soc Entomol Argent 69(3-4):233-252.

Molineri, C. 2010b.The influence of floods on the life history of dominant mayflies (Ephemeroptera) in a subtropical mountain stream. Studies on Neotropical Fauna and Environment 45(3):149-157.

Molineri, C., F. Romero, and H. R. Fernández. 2009. Diversidad y conservación de invertebrados acuáticos. En Brown, A. D., P. G. Blendinger, T. Lomáscolo y P. García Bes. Selva Pedemontana de Las Yungas. Historia Natural, Ecología y Manejo de un Ecosistema en Peligro. Ediciones del Subtrópico. Fundación Pro-Yungas. San Miguel de Tucumán, Tucumán, Argentina.

Morrone, J. J. 2004. La zona transición sudamericana: Caracterización y relevancia evolutiva. Acta Ent Chilena 28(1): 41-50.

Nieto, C., D. A. Dos Santos, A. E. Izquierdo, J. S. Rodríguez, and H. R. Grau. 2017. Modelling beta diversity of aquatic macroinvertebrates in High Andean wetlands. Journal of Limnology 76:155-170. doi: 10.4081/jlimnol.2017.1600.

Oksanen, J. F., G. Blanchet, M. Friendly, R. Kindt, P. Legendre, D. McGlinn, P. R. Minchin, R. B. O'Hara, G. L. Simpson, P. M. Solymos, H. H. Stevens, E. Szoecs, and H. Wagner. 2016. vegan: Community Ecology Package. R package version 2.4-0. URL: CRAN.R-project.org/package=vegan. 
Parra, L. E., and J. A. Morales. 2003. Descripción e interpretación de la columna estratigráfica tipo de la serie vulcanosedimentaria neógena del Valle del Cajón (Catamarca, Argentina). Geogaceta 33:55-58.

Pave, J. P., and M. Marchese. 2005. Invertebrados bentónicos como indicadores de calidad del agua en ríos urbanos (Paraná-Entre Ríos, Argentina). Ecología Austral 15:183-197.

Pizzolón, L., and M. L. Miserendino. 2001. The performance of two regional biotic indices for running water quality in Northern Patagonian Andes. Acta Limnologica Brasiliensia 13:11-27.

Pessacq, P., and M. L. Miserendino. 2008. Ephemeroptera and Plecoptera biodiversity in central Patagonia, Chubut province, Argentina. Zootaxa 1817:27-38.

Prat, N., B. Ríos, R. Acosta, and M. Rieradevall. 2009. Los macroinvertebrados como indicadores de calidad de las aguas. Pp. 631-654 en Macroinvertebrados Bentónicos Sudamericanos. Sistemática y Biología. Domínguez, E. y H. R. Fernández (eds.). Fundación Miguel Lillo. San Miguel de Tucumán, Tucumán, Argentina.

R Core Team. 2016. R: A language and environment for statistical computing. R Foundation for Statistical Computing, Vienna, Austria. URL: www.R-project.org.

Romero, V. F., H. R. Fernández, V. Manzo, C. Molineri, M. Correa, and M. C. Nieto. 2011. Estudio integral de la cuenca del Río Lules (Tucumán): aspectos biológicos. Pp.111-135 en Fernández, H. R. y H. M. Barber (eds.). La Cuenca del río Lules. Una aproximación multidisciplinaria a su complejidad. EDUNT. San Miguel de Tucumán, Tucumán, Argentina.

Rosa, H. 2000. El Clima de La Rioja. En Catálogo De Recursos Humanos e Información Relacionada con la Temática Ambiental en la Región Andina Argentina. URL: goo.gl/MFkR8v.

Salcedo Gustavson, S. A., and F. A Trama. 2014. Manual de identificación de macroinvertebrados acuáticos de la microcuenca San Alberto, Provincia de Oxapampa, Perú. Rizo Patrón, F. L. (ed.). Chanchamayo, Perú.

Scheibler, E. E., and G. O. Debandi. 2008. Spatial and temporal patterns in the aquatic insect community of a high altitude Andean stream (Mendoza, Argentina). Aquatic Insects 30(2):145-161.

Scheibler, E. E., V. Pozo, and A. C. Paggi. 2008. Distribución espacio-temporal de larvas de Chironomidae (Diptera) en un arroyo andino (Uspallata, Mendoza, Argentina). Rev Soc Entomol Argent 67(3-4):45-58.

Tejerina, E., and C. Molineri. 2007. Comunidades de Chironomidae (Diptera) en arroyos de montaña del NOA: comparación entre Yungas y Monte. Rev Soc Entomol Argent 66(3-4):169- 177.

Vallanía, E. A., P. A. Garelis, E. S. Trípole, and M. A. Gil. 1996. Un índice biótico para las sierras de San Luis (Argentina). Rev UNRC 16(2):129-136.

Welcome, R. I. 1985. River Fisheries. FAO. Fisheries Technical Paper. Pp. 262-330.

Zelarayán Medina, G. F., and L. B. Salas. 2014. Estructura y variación espacial de las colectividades de artrópodos en el Río "Las Juntas" (Catamarca, Argentina). Huayllu-Bios 8:15-39 